\title{
Positive challenges for research in the changing Mediterranean environment
}

\author{
Wolfgang Cramer ${ }^{1}$
}

Published online: 10 October 2017

(C) Springer International Publishing AG 2017

The Mediterranean Basin, consisting of both the ocean and the surrounding land, is one of the richest places on Earth in terms of its diversity, culture and also its economy. Yet its environmental problems are staggering. Depending on how wide the littoral zone is considered to be, several hundred million people live on its shores today. Our ancestors have lived there for millennia, creating one of the cradles of modern civilization. Historians have speculated about the reasons for this remarkable coevolution, but the varied, rich and stable physical environment has likely played an important role in the development of human society. Throughout modern history, people have modified this environment, by developing landscapes through agriculture, gardening and settlements, but also by inflicting periods of degradation due to unsustainable use of the land or the sea. The associated damage has impacted human well-being, often due to the loss of direct "provisioning ecosystem services", such as food and fibre from agriculture, animal husbandry, forestry or fisheries.

Arguably, the degradation has never been more profound, and the consequences for the last remaining seminatural ecosystems are massive. At the same time, the current loss of natural capital for human development is unprecedented. In addition, the basin itself undergoes changes: rivers change their sediment load due to dams and other flow modifications, coastlines and in particular deltas and their wetlands change. The accelerating global

Wolfgang Cramer

wolfgang.cramer@imbe.fr

1 Institut Méditerranéen de Biodiversité et d'Ecologie marine et continentale (IMBE), Aix Marseille Université, CNRS, IRD, Avignon Université, Technopôle Arbois-Méditerranée, Bât. Villemin, BP 80, 13545 Aix-en-Provence Cedex 04, France warming, fuelled as it is also by Mediterranean emissions from burning fossil fuels, is beginning to drive a significant rise in sea levels. The Mediterranean coasts face a unique level of risk, resulting from a combination of large human settlements and other infrastructures very near current sealevel, and no protection from the consequences of Antarctic ice loss. A large number of these installations are in countries with large coastal cities and relatively low incomes. On top of the physical and biological consequences of environmental change come two largely independent human activities: the almost unmitigated air and water pollution from transport, industry and other sources, and the growing instability of political systems, including war and the flow of thousands of refugees with its associated terrible death tolls.

Given these trends, all of them intolerable from any human perspective, and all negative-from the loss of human life, to a growing disparity between the rich and the poor, the loss of marine and terrestrial biodiversity and the long-term pollution of the environment - can we really speak about "positive challenges"? One possible answer concerns scientific research and the content of contributions to the Euro-Mediterranean Journal for Environmental Integration, and similar publications.

It is reasonable to ask whether science, itself originating from the Mediterranean Basin (at least in its world-dominating Aristotelian form), could be harnessed more effectively to assist humanity in its efforts to achieve its fundamental objectives of equitable and sustainable development? The number of scientists and engineers from numerous disciplines who dedicate their work to the solution of environmental problems is huge. Thousands of researchers are working under the explicit assumption that a better understanding of processes such as biodiversity loss, chemical pollution, or changing water availability will 
help counter degradation. Such knowledge could certainly lead to better adaptation to the underlying trends, e.g., through the development of water purification methods or more efficient water use. Research findings can also contribute to mitigating the drivers of change, such as the reduction of greenhouse gas emissions or pollutants, or implementing more resource-efficient production methods. Many such studies are appearing in this and other journals and they will be valuable for those seeking ways to halt, or slow down, environmental degradation.

The role a scholarly scientific journal plays in this context merits being stated explicitly: without the filters provided by peer review, researchers are unlikely to attain the same level of precision, clarity and plausibility of their findings. Of course, this does not in itself guarantee "truth" for every statement being made, but the procedures of editing and reviewing journal papers are successful in improving the quality and usefulness of the output. And organising journals around topics such as "environmental integration" will make it more likely that research findings are noted by those who can use them.

But the positive challenges for science do not stop with the publication of papers on highly specific issues. The next level is to ask more fundamental questions, and to debate them. One such question could be, for example, to seek a more concrete meaning of the term "environmental integration". What kind of knowledge needs to be integrated? And how can it be done? Reflecting about this leads to interesting intellectual challenges, but it is important to note that the quest for more sustainable development of the (Mediterranean) environment also depends on it directly. Tracing back, for example, from the degradation of land and sea use systems to the underlying social, economic and political processes clearly implies environmental integration. Engaging in such studies can, therefore, be considered a positive challenge. If successful, such work could contribute even more directly to the development of sustainable pathways for the Mediterranean environment and the people depending on it.

Integrative studies require many scientists to work together, bringing not only different disciplines but also different personal experiences into the team. This is where perhaps the most promising positive challenge in the Mediterranean exists: scientific research is being pursued in all countries around the basin, but with widely different traditions, languages and knowledge bases. Bringing these together can help solve concrete scientific problems that could not be addressed by only a small team in some place. But an important additional effect is that such collaboration also fosters personal and social networks, across the boundaries of countries and world views. It is no exaggeration to claim that environmental integration, expressed through suitable scientific studies pursued by international and interdisciplinary teams, can create better understanding between societies and hence reduce conflict.

Fostering pan-Mediterranean interdisciplinary studies can be seen as the real meaning of environmental integration. A scientific journal cannot create these kinds of dynamics. But its authors, reviewers and editors can encourage work to be done in truly integrative contexts, and there is no doubt that the founders of the EuroMediterranean Journal for Environmental Integration had exactly this ambition. It is now up to the scientific community to use the platform, for the greater benefit of all people around the Mediterranean. 